\title{
Automated Discovery in Elementary Extrema Problems
}

\author{
Francisco Botana* and José L. Valcarce \\ Departamento de Matemática Aplicada I, Universidad de Vigo, Campus A \\ Xunqueira, 36005 Pontevedra, Spain \\ fbotana@uvigo.es \\ Departamento de Matemáticas, IES Pontepedriña, 15702 Santiago, Spain \\ jvalcarce@edu. xunta.es
}

\begin{abstract}
This paper describes an enhancement of GDI, a dynamic geometry environment that uses symbolic techniques for performing automatic proof and discovery in elementary geometry. We report a successful add-on that automatically discovers critical points in elementary extrema problems specified via diagrams. Based in classical one variable calculus, the new module, Optimus, is a step ahead in the cooperation between dynamic geometry and computer algebra systems. It can be used in technology rich environments for mathematics education, adding new calculus abilities to dynamic geometry software.
\end{abstract}

\section{Introduction}

Dynamic geometry software refers to computer programs where accurate construction of geometric configurations can be done. The key characteristic of this software is that unconstrained parts of the construction can be moved and, as they do, all other elements automatically self-adjust, preserving all dependent relationships and constraints. Commercial versions of dynamic geometry environments, namely Cabri Geometry [9] and The Geometer's Sketchpad [8], appeared ending the 80's, and, since they experienced a fast and wide dissemination in computer aided mathematical education, remain today as market dominant tools. Nevertheless, their approach heavily relies on numerical strategies that have originated some problems which are creating a real bottleneck for future developments: the so called continuity problem, the loci generation and the proof capabilities [5].

The continuity problems refers to dynamic diagrams where a small move of an element causes a sudden jump of a dependent element. It appears in constructions where a dependent point is located on a intersection comprising at least a quadratic object.

The loci generation problem deals with the way geometric loci are computed in standard dynamic geometry. The sampling approach taken in most systems

\footnotetext{
* Partly supported by grant MTM2004-03175 from the Spanish MEC and by the

Technical University of Eindhoven, as a visiting scholar during March 2004.
} 
only allows the generation of parametrically defined loci, and their equations are also unknown [2].

Regarding proof abilities, it must be noted that the accuracy of the constructions and the possibility to experiment with different instances of them have led to the extensive use of an activity that has been termed as 'visual proving' (of properties and theorems). Furthermore, Cabri incorporates an (unexplained) 'property checker' that can give an answer to a reduced list of geometric statements about a diagram.

Partly reacting to this numerical approach last generation dynamic geometry programs such as Cinderella [1], GDI [1, 3, 4, GEOTHER [13 and GEX 7] start including formal tools. Based on projective geometry and Cayley-Klein geometries (Cinderella), on the Groebner bases method (GDI), or on Ritt-Wu characteristic sets (GEX and GEOTHER), these systems connect dynamic geometry with solid mathematical theories. Besides that, GDI and GEOTHER show up two successful examples of cooperation between an interactive environment and a computer algebra system (Mathematica and CoCoA [6] in GDI, Maple in GEOTHER).

This simple note describes a new way for computing, in an automatic manner, critical points in elementary extrema points described through dynamic diagrams. Section 2 states the problem and illustrates the Optimus module by means of a single exercise. The limitations and some extensions of the approach are discussed in Section 3. Section 4 deals with a possible connection of Optimus with other dynamic geometry environments via OpenMath.

\section{User Interface and Architecture}

We will restrict the study to optimization problems in one variable. Furthermore, we will only consider problems that admit a plane geometric modelization in a dynamic geometry setting, where the magnitude to optimize is defined by means of a dependent point, or can be reduced to it.

Let us illustrate the use of Optimus with a simple case: Two vertical poles $O A$ and $P B$ are secured by a rope $A X B$ going from the top $A$ of the first pole to a point $X$ on the ground between the poles and then to the top $B$ of the second pole. If the length of poles are 1 and 2 , find the position of $X$ that requires the least rope.

The first task consists of building a dynamic model of the problem (Fig. 1). Note that, besides the coordinate axes based on points $O(0,0)$ and $U(1,0)$ (being $U$ hidden in the diagram), there are only three free points $A(0,1), P(2,0), B(2,2)$ and a dependent point $X\left(x_{1}, x_{2}\right)$, which lies on the line passing through $O$ and $U$. Since GDI uses a coordinate-based approach, the whole construction can be algebraically described by the coordinates of points and the condition $x_{2}=0$. Once the construction is done, the user must specify the magnitude to optimize and the point on which it depends through an ad-hoc designed window (Fig. 2). The magnitude distance $(A, X)+\operatorname{distance}(X, B)$ is internally translated as the sum of a pair of polynomial constraints $x_{3}+x_{4}$, whose definitions are 


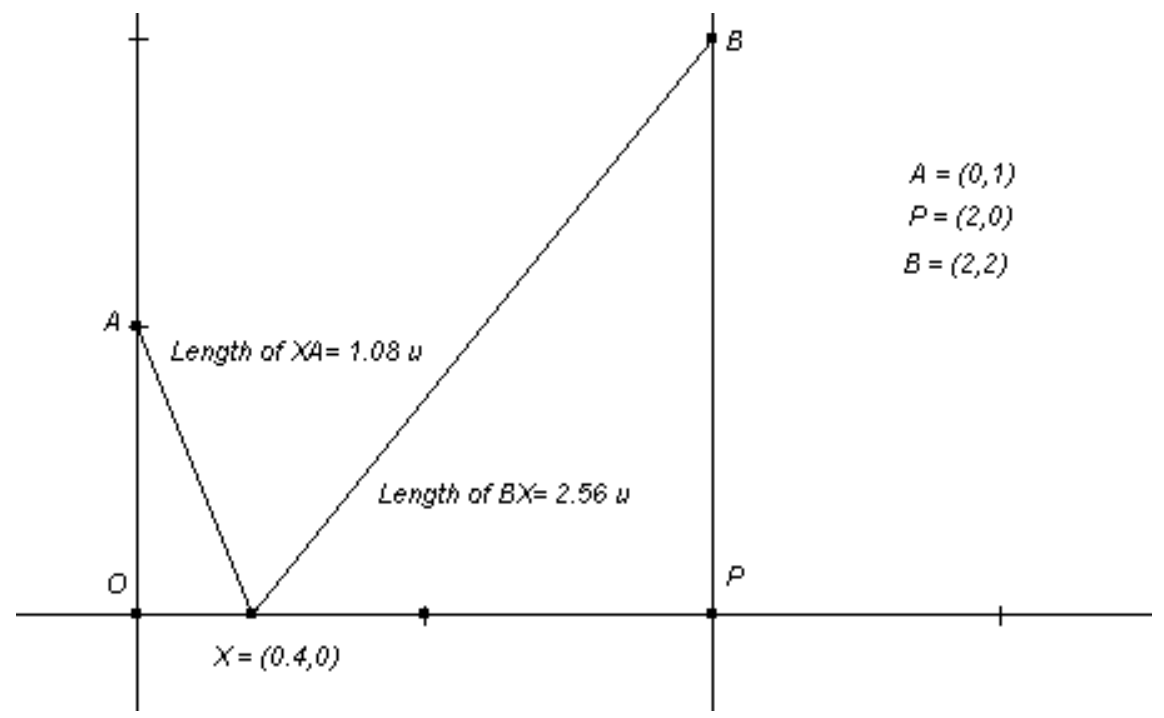

Fig. 1. The construction for the two poles problem

$x_{3}^{2}=\left(x_{1}^{2}+\left(1-x_{2}\right)^{2}\right)$ and $x_{4}^{2}=\left(\left(2-x_{1}\right)^{2}+\left(2-x_{2}\right)^{2}\right)$. Although taking the square distances will be a source of imprecision, the need of coherence with other GDI agents (prover, discoverer, ...) compelled us to maintain the computations in a strict algebraic environment.

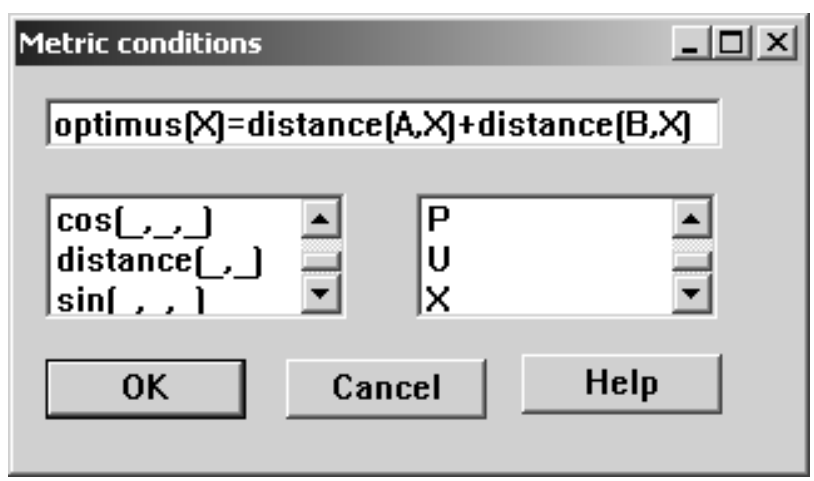

Fig. 2. Specifying the point and the magnitude to optimize

Once given the preceding information, GDI launches a local copy of Mathematica, if any, or calls a remote kernel via webMathematica. A small Mathematica package then performs the following operations:

- read the construction points and constraints and the optimization items,

- solve the polynomial constraints $\left(x_{2} \rightarrow 0\right)$, 
- solve the optimization constraints $\left(x_{3} \rightarrow \pm \sqrt{x_{1}^{2}+\left(1-x_{2}\right)^{2}}, x_{4} \rightarrow \pm \sqrt{\left(2-x_{1}\right)^{2}+\left(2-x_{2}\right)^{2}}\right)$,

- for each combination of the preceding solutions

- solve the derivative of the optimization condition with respect to the unbound variable of the optimization point $\left(x_{1}\right)$,

- compute the coordinates of the construction dependent points,

- add this set of points to a list of possible solutions,

- return this list.

The list of critical and other dependent points is shown as a window in the main screen of GDI or, if a remote Mathematica kernel is being used, by means of a browser (Fig. 3). A numerical approximation of the coordinates is also given in order to help the student placing the points in the dynamic construction. Note also that, of course, the first point $X(-2,0)$ does not optimize the length of the rope, and it has been found due to the polynomial restriction above mentioned.

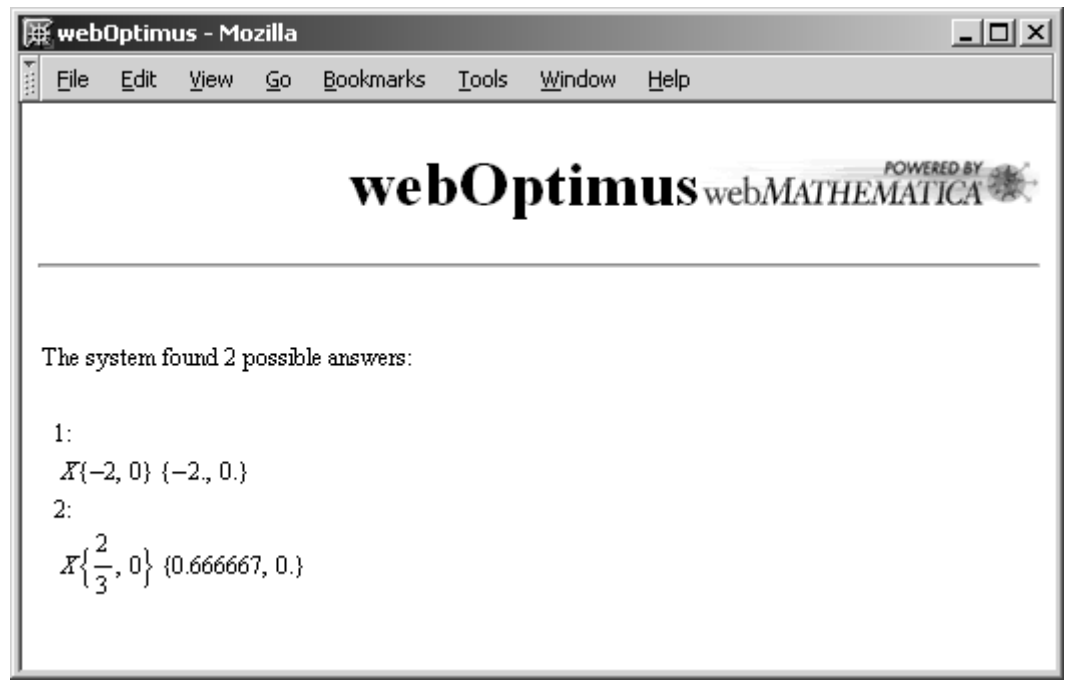

Fig. 3. The critical points found by the system

\section{Limitations and Extensions of the Approach}

Apart from the introduction of spurious solutions due to the polynomial restriction, it must be noted that the approach is not complete. Furthermore, it is recommended that the system will be used under teacher guidance in elementary levels, since it sometimes poses subtle questions requiring mature mathematical skills or a deep knowledge of the system design.

Consider, for instance, finding which isosceles triangle inscribed in a given circle has the greatest area. A common behavior in a dynamic geometry setting would be drawing a circle and placing two points $A\left(x_{1}, x_{2}\right)$ and $X\left(x_{3}, x_{4}\right)$ on the circle. The third triangle vertex $B\left(x_{7}, x_{8}\right)$ can be easily constructed through 
the perpendicular bisector of segment $A X$ and its intersection with the circle. With this construction Mathematica finds 32 solutions to the set of polynomial constraints when solving respect to an unbound coordinate of $X$, say $x_{3}$. Eight of them are degenerate cases $(A=X)$, since they include the rules $x_{1} \rightarrow x_{3}$ and (taken with equal sign) $x_{2} \rightarrow \pm \sqrt{1-x_{3}^{2}}, x_{4} \rightarrow \pm \sqrt{1-x_{3}^{2}}$. So, point $B$ is ill-defined and no derivative is computed because the optimization condition is null. Other sixteen solutions have two degrees of freedom: no rules for $x_{1}, x_{3}$ are found, according to the fact that $A$ and $X$ have just been defined as arbitrary points on the circle. The triangle area is then a two-argument function, and the algorithm aborts the a posteriori computations. The remaining eight solutions include the rules $x_{1} \rightarrow x_{3}, x_{2} \rightarrow \pm \sqrt{1-x_{3}^{2}}, x_{4} \rightarrow \mp \sqrt{1-x_{3}^{2}}$, generating the four solutions returned by the system

$$
\begin{array}{r}
X(-1 / 2,-\sqrt{3} / 2), A(-1 / 2, \sqrt{3} / 2), B(1,0) \\
X(-1 / 2, \sqrt{3} / 2), A(-1 / 2,-\sqrt{3} / 2), B(1,0) \\
X(1 / 2,-\sqrt{3} / 2), A(1 / 2, \sqrt{3} / 2), B(-1,0) \\
X(1 / 2, \sqrt{3} / 2), A(1 / 2,-\sqrt{3} / 2), B(-1,0)
\end{array}
$$

The shown incompleteness could be avoided just reducing the construction freedom. Paying the price for some lost of generality, $A$ could be defined to be $U(1,0)$, being $X$ a point freely movable on the circle. The system returns in this case the complete set of solutions

$$
\begin{aligned}
& X(-1 / 2,-\sqrt{3} / 2), B(-1 / 2, \sqrt{3} / 2) \\
& X(-1 / 2, \sqrt{3} / 2), B(-1 / 2,-\sqrt{3} / 2)
\end{aligned}
$$

Note that a stronger connection of the Optimus and Discovery modules (not yet fully implemented) will allow a user to get an answer such as The isosceles triangle with greatest area inscribed in the circle must be equilateral (see [5] for the abilities of GDI finding geometric properties expressed with natural language statements).

The restriction of using Optimus only for one variable problems deserves additional explanation. GDI offers an option for specifying extra constraints to a construction. This option is the key for finding geometric loci with a posteriori conditions, or for discovering facts about essentially non constructive situations (see [10] for a paradigmatic example, the MacLane $8_{3}$ theorem). Through the introduction of extra constraints the system can be extended to deal with optimization problems in several variables. Consider finding the right triangle of given perimeter and maximum area: defining a vertex to be the origin $O$ and placing the remaining vertices $A, X$ as dependent points on both axes, the user can declare the perimeter to be a constant, say 14 ,

$$
\operatorname{distance}(O, X)+\operatorname{distance}(O, A)+\operatorname{distance}(A, X)=14
$$

and ask about the maximum area of the triangle, taking $X$ as the dependent point. Two of the eight found solutions are shown in Fig. 4, being the remaining 
ones symmetrical. Again, the consideration of signed distances leads up to the second group of solutions.

Another possible extension of Optimus has been studied. A construction involving an optimization condition can be done without any reference to numerical coordinates, that is, the coordinates of the free points or the values of the constants can be symbolic (as they are when using the proving abilities of GDI). In this case, the obtained solutions will exhibit more generality, but the price to pay is, in general, an unmanageable increment of the expressions of dependent points. So, since these values are not easily readable, we have restricted the domain of Optimus to constructions referred to a coordinate system.

4:

$$
X\{0,7(2-\sqrt{2})\}\{0 ., 4.10051\} A\left\{\frac{14(-7+7(2-\sqrt{2}))}{-14+7(2-\sqrt{2})}, 0\right\}\{4.10051,0 .\}
$$

8:

$$
X\{0,7(2+\sqrt{2})\}\{0 ., 23.8995\} \quad A\left\{\frac{14(-7+7(2+\sqrt{2}))}{-14+7(2+\sqrt{2})}, 0\right\}\{23.8995,0 .\}
$$

Fig. 4. A pair of solutions of the two variable problem

\section{Using Optimus with Other Dynamic Geometry Systems}

A goal of this short note consists of illustrating the connection of a dynamic geometry environment with a computer algebra system [12] for performing exact computations about optimization problems. Since the inputs to the algorithm described in Section 2 are the list of construction points, the constraints, a dependent point and the magnitude to optimize, any other dynamic geometry system could be easily modified to deal with such problems. Nevertheless, the character of proprietary software of most programs would convert this task in a time consuming work, unless developed by their own designers. The Geometer's Sketchpad, for instance, can export its constructions to html code, for being visualized using the JavaSketchpad applet [15. In this code, the value of an applet parameter contains a readable description of the construction. Similarly, Cabri has an option, Show Figure Description, that roughly describes a construction. Cinderella and GEX have also a mechanism to see the textual description of constructions. These tools can be used to analyse the construction and to get the list of points and constraints. A little piece of code could be then developed in order to select the point and optimization conditions and to launch Optimus. Nevertheless, in our opinion, a more promising future involves the use of a common protocol for intercommunication such as OpenMath [16] or the 
newest proposal GOOL [14. Using OpenMath and the experimental plangeo Content Dictionaries [17, a construction such as the one used in Section 2 can be described by

$$
\begin{gathered}
\operatorname{point}(O(0,0)), \operatorname{point}(U(1,0)), \text { point }(A(0,1)), \operatorname{point}(P(2,0)), \operatorname{point}(B(2,2)), \\
\text { line }(l, i n c i d e n t(O, l), \text { incident }(U, l)), \text { point }(X, \operatorname{incident}(l, X))
\end{gathered}
$$

and the optimization condition, defining a new OpenMath symbol optimus, by

$$
\operatorname{optimus}(X, \operatorname{distance}(A, X)+\operatorname{distance}(X, B)
$$

The first part of this OpenMath code can be easily translated into a set of points and a list of polynomials, as shown in the preceding Section. The optimization condition carries up the rest of the required information for Optimus. So, any OpenMath-capable system could connect to a remote Mathematica kernel for performing this kind of computations.

\section{Conclusion}

We extend a dynamic geometry environment with the ability of computing critical points in elementary one variable optimization problems diagrammatically described. To this end, we connect our dynamic tool with a computer algebra system (Mathematica), where standard calculus techniques are employed. Furthermore, this connection can be either local, either via a remote Mathematica kernel.

While optimization problems are usually managed by means of approximation techniques and empirical testing in educational uses of common dynamic geometry systems, our proposal gives exact solutions to the exercises and, being fully integrated with the geometry reasoning system, can be also exploited for proving or discovering tasks.

\section{References}

1. Botana, F., Valcarce, J.L.: A dynamic-symbolic interface for geometric theorem discovery. Computers and Education, 38(1-3), 21-35 (2002)

2. Botana, F.: Interactive versus symbolic approaches to plane loci generation in dynamic geometry environments. Proc. I Int. Workshop on Computer Graphics and Geometric Modeling (CGGM 2002), LNCS, 2330, 211-218 (2002)

3. Botana, F., Valcarce, J.L.: A software tool for the investigation of plane loci. Mathematics and Computers in Simulation, 61(2), 141-154 (2003)

4. Botana, F., Valcarce, J.L.: Automatic determination of envelopes and other derived curves within a graphic environment. Mathematics and Computers in Simulation, 67(1-2), 3-13 (2004)

5. Botana, F., Recio, T.: Towards solving the dynamic geometry bottleneck via a symbolic approach. Proc. 5th Int. Workshop on Automated Deduction in Geometry (ADG 2004), LNAI, 3763, 92-110 (2006) 
6. Capani, A., Niesi, G., Robbiano, L.: CoCoA, a system for doing Computations in Commutative Algebra. Available via anonymous ftp from: cocoa.dima.unige.it

7. Gao, X.S., Zhang, J.Z., Chou, S.C.: Geometry Expert. Nine Chapters, Taiwan (1998)

8. Jackiw, N.: The Geometer's Sketchpad v 4.0. Key Curriculum Press, Berkeley (2002)

9. Laborde, J. M., Bellemain, F.: Cabri Geometry II. Texas Instruments, Dallas (1998)

10. Recio, T., Botana, F.: Where the truth lies (in automatic geometry theorem proving). Proc. II Technical Session on Computer Graphics and Geometric Modeling (TSCG 2004), LNCS, 3044, 761-770 (2004)

11. Richter-Gebert, J., Kortenkamp, U.: The Interactive Geometry Software Cinderella. Springer, Berlin (1999)

12. Roanes-Lozano, E., Roanes-Macías, E., Villar, M.: A bridge between dynamic geometry and computer algebra. Mathematical and Computer Modelling, 37(9-10), 1005-1028 (2003).

13. Wang, D.: GEOTHER: A geometry theorem prover. Proc. 13th International Conference on Automated Deduction (CADE 1996), LNCS, 1104, 166-170 (1996)

14. Wang, D., Liang, T.: Towards a Geometric-Object-Oriented Language. Proc. 5th Int. Workshop on Automated Deduction in Geometry (ADG 2004), LNAI, 3763, 130-155 (2006)

15. http://www.keypress.com/sketchpad/javasketchpad/

16. http://www.openmath.org

17. http://www.win.tue.nl/ ${ }^{\sim}$ amc/oz/om/cds/geometry.html 